\title{
Supplementary enteral nutrition maintains remission in paediatric Crohn's disease
}

\author{
M Wilschanski, P Sherman, P Pencharz, L Davis, M Corey, A Griffiths
}

\begin{abstract}
Background-Liquid diets given enterally combined with 'bowel rest' are efficacious in the treatment of active Crohn's disease, but rapid recrudescence of gastrointestinal symptoms after resumption of a normal diet is common.

Aims-This study examined whether continuation of enteral nutrition as a nocturnal supplement to an ad libitum daytime intake of a normal diet increased the length of remission of Crohn's disease in children.
\end{abstract}

Patients and methods-Children and adolescents with active Crohn's disease treated successfully with exclusive enteral nutrition were classified retrospectively according to whether they continued supplementary enteral nutrition or not. Time to relapse and linear growth were compared between the two cohorts.

Results-Between January 1986 and December 1992, 65 patients aged 7-17 years (mean (SD) $13.6(2.1)$ years) (36 males, 29 females) with Crohn's disease in exacerbation were treated for $\geqslant$ four weeks by bowel rest and nasogastric tube feeding of an oligopeptide or amino acid based formula. At first follow up visit, remission (fall in Paediatric Crohn's Disease Activity Index, PCDAI to $\leqslant 20$ ) was achieved in 47 of $65(72 \%)$ patients. Subsequently, 20 of these $47(43 \%)$ relapsed by six months and 28 of $47(60 \%)$ by 12 months. Patients who continued nasogastric supplementary feeding $(n=28)$ after resumption of an otherwise normal diet remained well longer than those who discontinued nocturnal supplements completely $(n=19) \quad(p<0 \cdot 02)$. Furthermore, continued use of nasogastric supplements before completion of puberty was associated with improved linear growth.

Conclusion-After successful treatment of active Crohn's disease by exclusive enteral nutrition, supplementary enteral nutrition without restriction of normal diet is associated with prolongation of remission and improved linear growth in children and adolescents.

(Gut 1996; 38: 543-548)

Keywords: paediatric Crohn's disease, nutrition treatment, growth.

Corticosteroids remain the most effective available medical treatment for acute exacerbations of Crohn's disease. ${ }^{2}$ Their use in the paediatric population is limited by the adverse effect of longterm daily use on linear growth. Therefore, alternative treatments have been sought. Exclusive enteral nutrition using formulated food has been increasingly used as primary treatment of active Crohn's disease. ${ }^{34}$ Efficacy has been shown in controlled clinical trials, but symptoms recur in a high percentage of patients within several months of stopping the diet treatment. ${ }^{5}$ Longterm exclusive enteral nutrition and avoidance of a normal diet is an unreasonable therapeutic option. Compliance is much better, however, when normal food is permitted during the day. Such supplementary nocturnal nasogastric tube feeding is often used in children as a means of improving growth. ${ }^{6}$ We have found that patients using such supplementary feeding regimens also experience fewer relapses of gastrointestinal symptoms. To examine this question formally, we reviewed our experience with enteral nutrition as primary treatment of active Crohn's disease in children and adolescents. We designed a historical cohort study to discover if continued supplementary enteral nutrition without other dietary restrictions is associated with prolongation of remission.

\section{Methods}

\section{Patients}

The medical records of all patients with active Crohn's disease treated by exclusive nasogastric tube feeding of an elemental or semielemental liquid diet at the Hospital for Sick Children, Toronto, between January 1986 and December 1992 were reviewed. The formula used varied according to the time period, but otherwise all patients were treated with an identical protocol as outlined below.

Patients were admitted to hospital to learn the techniques of nasogastric tube insertion and formula infusion. Infusion rates of formulated food were increased in a stepwise fashion to provide the recommended dietary allowance $(\mathrm{RDA})^{7}$ of total energy and protein during a 10 to 14 hours overnight infusion. Only clear fluids were permitted by mouth during the day. Patients were discharged from hospital when comfortable with the regimen, usually after five to seven days. Exclusive enteral nutrition was then continued at home. The child removed the feeding tube each morning to facilitate normal daytime activities and reinserted it each evening. Oral corticosteroids being given at the start of the liquid diet treatment were tapered and discontinued. Other medications, with the exception of metronidazole for perianal disease, were discontinued. In all patients 
TABLE I Baseline characteristics of the patients

\begin{tabular}{ll}
\hline Age & $13 \cdot 6(2 \cdot 1)$ years (range $7-17)$ \\
Sex & 36 males, 29 females \\
Anatomical localisation of disease & 27 \\
Small intestinal only (includes ileal \pm caecal) & 33 \\
Ileocolonic disease & 5 \\
Colon only & $2 \cdot 0(2 \cdot 0)$ years (range $0 \cdot 2-12)$ \\
Duration of diagnosed disease & $(9$ newly diagnosed; 56 relapsed patients) \\
& I 26, II 17, III 7, IV $6, \mathrm{~V} 9$ \\
Tanner stage: & $25(9$ patients in first exacerbation) \\
Concurrent medications at baseline & 13 \\
None & 13 \\
Sulphasalazine or 5 -aminosalicylic acid only & 12 \\
Metronidazole & 13 \\
Prednisone $(<0.5 \mathrm{mg} / \mathrm{kg} /$ day $)$ & $(10$ patients receiving $\geqslant 2$ drugs $)$ \\
Prednisone $(>0.5 \mathrm{mg} / \mathrm{kg} /$ day) &
\end{tabular}

Data shown as mean (SD).

anatomic localisation of disease had been recorded by radiological examination of the small intestine and radiological or endoscopic examination of the colon, or both. Duration of disease before the start of treatment and concurrent medications were recorded. Baseline measurements at treatment initiation included age, sex, height, weight, and Tanner stage.

After the first follow up visit, all patients who had achieved remission with exclusive enteral feeding resumed a normal an libitum daytime diet. Patients were encouraged by the consulting gastroenterologist, however, to supplement their daily intake of normal food with a lesser amount of the same liquid diet (usually $50-60 \%$ of the initial nightly amount) as a possible means of maintaining clinical remission of Crohn's disease. This was given through a nasogastric tube four to five nights weekly. Patients with growth potential were advised that such supplements could facilitate growth. ${ }^{6}$

For this study patients were classified retrospectively into two cohorts according to whether or not they chose to continue the nocturnal supplements after successful induction of clinical remission. Time to relapse and subsequent linear growth of patients belonging to the supplementary enteral nutrition cohort were compared with those in the control cohort. All heights were measured on a wallmounted stadiometer.

\section{Assessment of disease activity and response to treatment}

Activity of Crohn's disease was assessed clinically at the start of treatment and at first follow up visit using the Paediatric Crohn's Activity Index (PCDAI), a multi-item score comprising clinical symptoms as well as packed cell volume, erythrocyte sedimentation rate (ESR), and albumin. ${ }^{8}$ Other laboratory parameters, including haemoglobin and serum orosomucoid, were also measured sequentially.

TABLE II Clinical and laboratory parameters at baseline and first follow up

\begin{tabular}{lccc}
\hline & Baseline & First follow up & p Value \\
\hline Body weight (kg) & $36 \cdot 5(11 \cdot 8)$ & $42 \cdot 1(11 \cdot 7)$ & $<0 \cdot 0001$ \\
PCDAI & $40 \cdot 3(11 \cdot 8)$ & $15 \cdot 4(14 \cdot 5)$ & $<0 \cdot 001$ \\
Packed cell volume & $34 \cdot 8(4 \cdot 3)$ & $36 \cdot 1(3 \cdot 3)$ & $0 \cdot 02$ \\
Haemoglobin $(\mathrm{g} / \mathrm{l})$ & $113(15 \cdot 1)$ & $118 \cdot 4(11 \cdot 6)$ & $0 \cdot 003$ \\
ESR (mm in first hour) & $28 \cdot 2(19 \cdot 8)$ & $22 \cdot 6(19)$ & $0 \cdot 05$ \\
Orosomucoid $(\mathrm{g} / \mathrm{l})$ & $1 \cdot 43(0 \cdot 5)$ & $1 \cdot 04(0 \cdot 5)$ & $<0 \cdot 0001$ \\
Albumin $(\mathrm{g} / \mathrm{l})$ & $32 \cdot 6(4)$ & $36 \cdot 6(4 \cdot 6)$ & $<0 \cdot 0001$ \\
\hline
\end{tabular}

Data shown as mean (SD).
Success of exclusive enteral nutrition was defined as a decline in PCDAI to $\leqslant 20$ at first follow up. Treatment failure was defined as the PCDAI remaining $>20$ or the patient being unable to tolerate the feeding regimen. Among responders relapse was defined by return of clinical symptoms necessitating additional treatment and associated rise in PCDAI to $>20$.

\section{Assessment of patient acceptance}

A questionnaire was given to all children who had received exclusive enteral nutrition as primary treatment. Patients were asked to report adverse effects of nasogastric tube feeding and were questioned specifically about night-time wakening, nausea, vomiting, abdominal cramping, and loose stools. Patients who had previously been treated with oral corticosteroids were asked to state which, if either, of the two treatments they preferred. The questionnaire received the prior approval of The Human Subjects Review Committee at the Hospital for Sick Children, Toronto.

\section{Statistical analysis}

All data are expressed as mean (SD). Baseline values for PCDAI, age, and disease duration in the two cohorts were compared using the Student's $t$ test. PCDAI and laboratory parameters at baseline and at first follow up visit and linear growth during the years before and after exclusive enteral nutrition were compared by the paired Student's $t$ test. Comparisons of different anatomical locations were performed using two tailed Fisher's exact test. Rates of clinical relapse were compared using the log rank procedure. Changes in height velocity in the two groups were compared using the Student's $t$ test.

\section{Results}

Between January 1986 and December 1992, 65 children and adolescents received exclusive elemental or semi-elemental liquid diets by nocturnal nasogastric infusion to treat active Crohn's disease. The formulated food used was either Vital HN (Abbott Laboratories, $n=51$ ), Vivonex (Sandoz Nutrition, $n=8$ ), or Peptamen (Clintec Nutrition, $n=6$ ). Table I summarises the characteristics at baseline. The study excluded an additional seven growth impaired patients without overt symptoms of intestinal inflammation, who were treated with enteral nutrition as a supplement to a normal diet but without an initial period of bowel rest. Another two patients with active Crohn's disease given with liquid diet treatment combined with bowel rest were excluded because immunosuppressive drugs were started simultaneously and may have contributed to disease control.

The PCDAI had fallen to $<20$ in 47 of 65 children $(72 \%)$ at first follow up examination $1.5(0.4)$ months (range $0.5-2.5$ months) after the start of exclusive enteral nutrition. Of the 18 treatment failures, only three were 


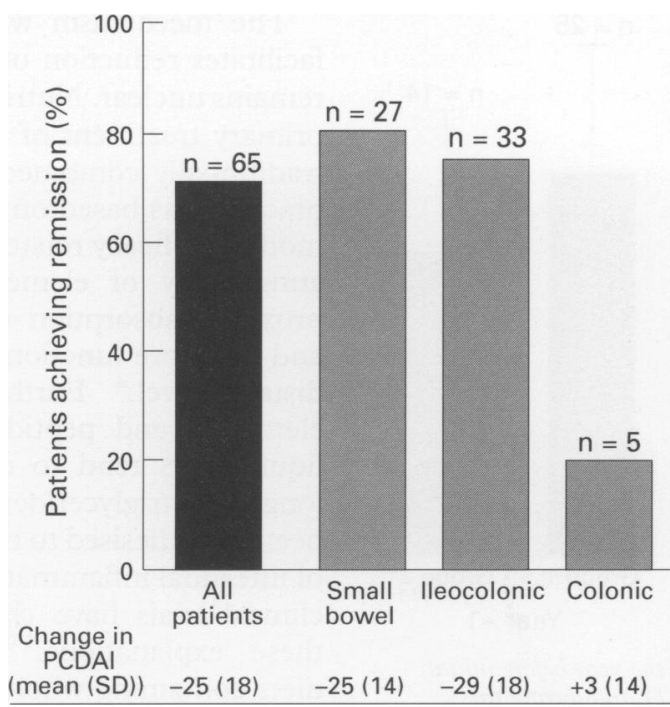

Figure 1: Percentage of patients achieving remission with exclusive enteral nutrition according to anatomical localisation of disease.

attributable to intolerance to or non-compliance with the nasogastric tube feeding. Table II shows mean values for PCDAI, weight, and laboratory parameters for all 65 patients at initial and first follow up assessments. As shown in Fig 1, fewer patients with isolated colonic Crohn's disease achieved clinical remission (one of five, 20\%) compared with the other anatomical sites $(p<0.03)$.

Of the 47 patients who achieved remission, $20(43 \%)$ children had relapsed by six months and $28(60 \%)$ by 12 months. Those continuing nocturnal supplements $(n=28)$ did not differ significantly from those declining to do so $(n=19)$ with respect to age, site or duration of Crohn's disease, PCDAI at baseline or growth parameters. As Fig 2 shows, however, the cumulative probability of maintaining clinical remission was greater when supplementary feeding was continued during the year after

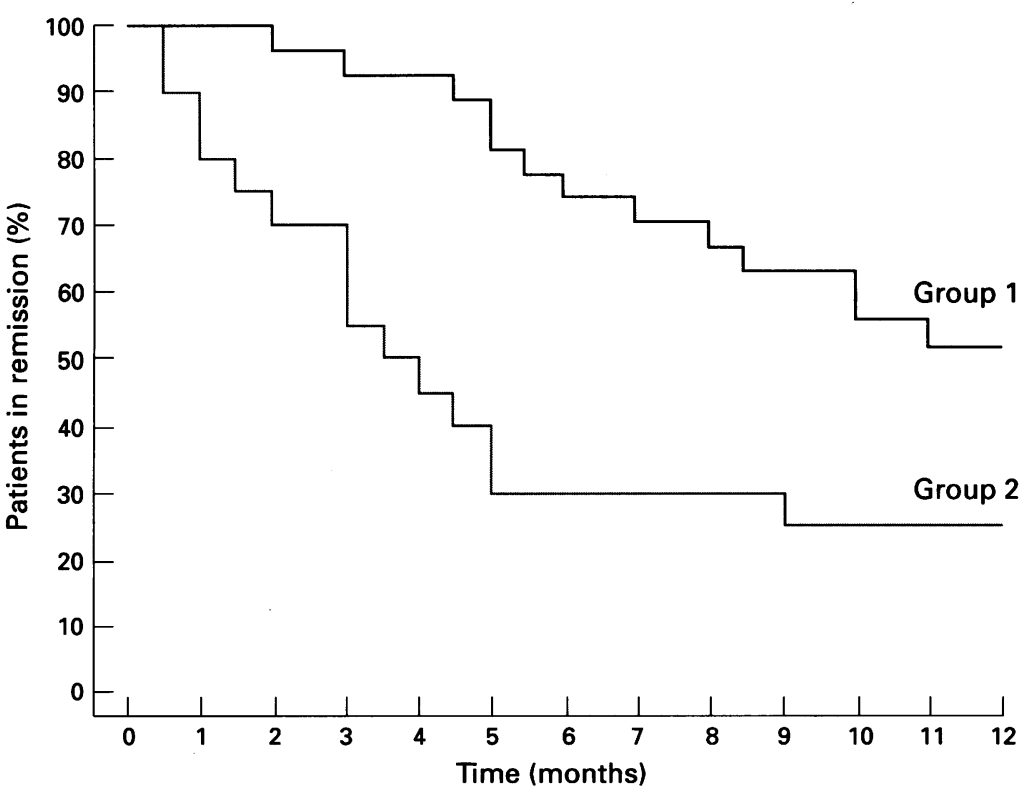

Figure 2: Cumulative percentages of patients remaining in clinical remission after resumption of ad libitum daytime diet. Group 1: cohort with nocturnal nasogastric liquid diet supplements $(n=28)$, group 2: cohort without supplements $(n=19)$ (log rank $p=0.005$ ) exclusive enteral nutrition. Relapse rates at both six months (15 of $19 v$ five of 28 , $\mathrm{p}<0.001$ ), and 12 months ( 15 of $19 v 12$ of 28 , $\mathrm{p}<0.02$ ) were higher in the control cohort compared with the cohort receiving nocturnal supplements.

One patient who continued supplementary feeds and two patients who did not were Tanner stage 5 and had completed their linear growth before treatment with enteral nutrition. Height velocity of the remaining patients with linear growth potential is displayed in Fig 3 for the year after initial administration of formulated food and the preceding year. Reliable height data from the year before treatment were lacking for newly diagnosed patients $(n=2$ in supplemented cohort, $n=6$ in control cohort) and for one other non-supplemented patient with Crohn's disease of long duration previously treated elsewhere. One control group patient emigrated temporarily so that height at 12 months could not be recorded. Follow up growth data from another two patients in each cohort were censored, because all four had intestinal resection at time of symptomatic relapse within the year after treatment with exclusive enteral nutrition. Hence change in linear growth velocity could not be attributed solely to nutritional intervention. The mean height velocity of the remaining 24 patients receiving supplementation with complete before and after treatment data was greater during the treatment year (6.1 (4.2 $\mathrm{cm})$ ) than during the previous year $(3 \cdot 2(1 \cdot 6$ $\mathrm{cm}))(p<0.001)$. For the seven non-supplemented patients with complete before and after treatment measurements, the mean height velocity during the second year ( $4 \cdot 2$ (4.5 $\mathrm{cm})$ ) did not differ significantly from that recorded during the previous year (3.8 (1.2 $\mathrm{cm})$ ). Comparing paired data between the two cohorts, the mean change in height velocity was $2.87 \mathrm{~cm} /$ year among those continuing supplements versus $0.4 \mathrm{~cm} /$ year among those who did not $(p=0.057)$.

\section{Results of questionnaire}

Questionnaires concerning acceptance of enteral nutrition were returned by 52 of 65 patients $(80 \%)$. As Table III shows, untoward symptoms including sleep disturbance and gastrointestinal complaints were common. Nevertheless, of 44 patients who had experience with both oral corticosteroid treatment and enteral nutrition, $20(45 \%)$ reported a preference of liquid diet treatment, whereas, $12(27 \%)$ had no preference and $12(27 \%)$ favoured the drug treatment.

\section{Discussion}

Active Crohn's disease successfully treated with exclusive enteral nutrition has been reported to relapse by one year after its discontinuation in 60 to $70 \%$ of patients. 5910 This was confirmed in our study among patients electing not to continue supplementary feeding. Our retrospective analysis suggests that continuation of liquid diet treatment as a 


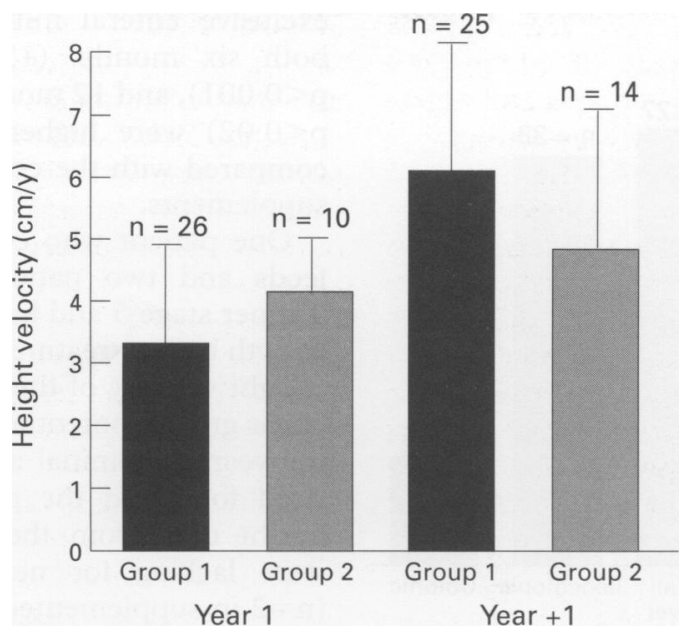

Figure 3: Height velocity during the year before initial exclusive enteral nutrition (year 1) and during the following year (year +1$)$. Bar graphs represent mean (SD). Patients $(n=3)$ whose linear growth was complete before short-term treatment with exclusive enteral nutrition are excluded. Group 1: patients with growth potential belonging to the supplementary nocturnal enteral nutrition cohort. Group 2: patients with growth potential belonging to the control cohort.

supplement to normal food, but without daytime dietary restrictions, prolongs the clinical remission induced by an initial period of exclusive enteral nutrition. The use of longterm dietary supplementation by nocturnal nasogastric infusion of formulated food to prevent relapse has not previously been reported. Harries et al ${ }^{11}$ reported a beneficial effect of oral dietary supplements in maintaining remission in a crossover trial among adults with Crohn's disease. We and others have found that children and adolescents will not consistently comply with recommendations for oral supplements. Therefore, nasogastric tube feeding has become more widely used. In a study of similar design to ours, Belli et al ${ }^{12}$ described improvement in clinical activity of Crohn's disease and growth among a much smaller group of children and adolescents who continued intermittent but exclusive enteral nutrition one month out of four over the course of one year, compared with those who declined to do so. The beneficial effects of such cyclical enteral nutrition combined with bowel rest on disease activity and growth were confirmed by Polk et al ${ }^{13}$ in an additional six patients who served as their own controls.

TABLE III Results of questionnaire (52 of 65 (80\%) returned)

\begin{tabular}{ll}
\hline & $\begin{array}{l}\text { Number (\%) of } \\
\text { patients reporting }\end{array}$ \\
\hline Night-time wakening & \\
Always & $11(21)$ \\
Often & $18(35)$ \\
Sometimes & $18(35)$ \\
Never & $2(4)$ \\
Abdominal cramps & $11(21)$ \\
Diarrhoea & $30(58)$ \\
Nausea & $23(44)$ \\
Vomiting & $22(42)$ \\
Patients with previous experience with & $\mathrm{n}=44$ \\
prednisone: & \\
Patients reporting preference for: & $20(45)$ \\
nutritional therapy (\%) & $12(27)$ \\
prednisone (\%) & $12(27)$ \\
\hline no preference (\%) & \\
\hline
\end{tabular}

The mechanism whereby enteral nutrition facilitates reduction of intestinal inflammation remains unclear. Nutritional treatments used as primary treatment of active disease have been traditionally combined with 'bowel rest'. This practice was based on the hypothesis that their mode of efficacy related either to the decreased antigenicity of elemental liquid diets or to proximal absorption of predigested nutrients and therefore functional bypass of the diseased distal bowel. ${ }^{4}$ Further, amino acid based elemental and peptide based semi-elemental liquid diets tend to contain low amounts of longchain triglycerides. A low fat content has been hypothesised to be necessary for reduction of intestinal inflammation. ${ }^{4}$ However, results of clinical trials have challenged the validity of these explanations. ${ }^{5914-16}$ Polymeric liquid diets containing whole protein and the normal $30-35 \%$ of energy as fat have been recently successfully used in the treatment of active Crohn's disease..$^{5}$ 14-16 $^{16}$ In andomised trial of adjunctive nutritional treatments, Greenberg et $a l^{17}$ found that partial parenteral nutrition plus an ad libitum oral diet was as effective in inducing clinical remission as either elemental liquid diets given by nasogastric tube or total parenteral nutrition and complete bowel rest among patients in hospital because of continuing activity of Crohn's disease despite high dose corticosteroid treatment. Furthermore, in a study comparing a semi-elemental with an elemental formula, the likelihood of attaining clinical remission correlated with an improvement in nutritional status rather than with effects on serum lipids. ${ }^{18}$ These findings support the hypothesis that the beneficial effect of enteral nutrition is primarily nutritional. It may depend on improved nutritional state in general or on the provision of an, as yet unidentified, micronutrient that facilitates healing of intestinal inflammation.

Our findings that supplementary enteral nutrition was associated with prolonged clinical remission despite resumption of regular food is consistent with a micro- or macro-nutritional effect, but not with the necessity of 'bowel rest' or avoidance of dietary antigenicity. Whether the type and amount of fat consumed by the supplementary enteral feeding group was significantly lower than that of the comparison group is not known. We did not ask patients in either group to keep daytime food diaries, and therefore cannot make definitive statements concerning the composition of their total dietary intake. The improved rate of weight gain and linear growth seen with supplementary feeding of an average 1250 calories five nights weekly, however, dictates that the unrestricted ad libitum oral intake was considerable.

Growth impairment is a common complication of childhood Crohn's disease. In our institution height velocity fell below the normal prepubertal rate $(\geqslant 4 \mathrm{~cm}$ per year) for at least two individual years and bone age was accordingly delayed in $50 \%$ of a group of 100 children who were at Tanner stage 1 or 2 of pubertal development at the time of diagnosis. ${ }^{19}$ The important mechanism of the observed 
retardation of growth is considered to be chronically inadequate intake of energy and protein resulting from both anorexia and aggravation of intestinal symptoms by eating. ${ }^{2021}$ Enteric protein leakage from the inflamed intestine, ${ }^{22}$ excessive losses of specific dietary nutrients such as zinc, corticosteroid inhibition of somatomedin production interact and may contribute to the significant growth delay in at risk children. ${ }^{23}$ Cytokines and other inflammatory mediators, such as tumour necrosis factor $\alpha$ (cachetin), may have a direct negative effect on both intake and utilisation of energy. ${ }^{24}$ Consistent provision of adequate nutrition either orally, enterally or parenterally is effective in restoring normal growth. ${ }^{6122021}$ Aiges et al previously reported linear growth improvement by supplementary nocturnal nasogastric tube feeding of formulated food in a comparison of children and adolescents complying with versus those refusing such treatment. ${ }^{6}$ We have confirmed this beneficial effect in a larger group of patients. The observed improvement in linear growth probably reflects improved nutritional intake, as well as control of intestinal inflammation without the use of daily corticosteroids. Greater use of nutritional treatment as alternative primary treatment of active Crohn's disease should reduce the frequency of growth impairment and improve the prognosis for ultimate height.

The 47 patients comprising the supplementary enteral nutrition and control cohorts in this study constituted $72 \%$ of all those in whom treatment of active Crohn's disease with exclusive enteral nutrition was attempted. This $72 \%$ rate of clinical response to exclusive enteral nutrition is very similar to the overall $78 \%$ response rate in a prospective, randomised, multicentre paediatric trial where the response rate among those treated with oral prednisone was $90 \% .{ }^{25}$ Exclusive enteral nutrition in other randomised controlled trials versus corticosteroids successfully induced remission as defined by clinical multi-item measures in $53 \%$ to $82 \%$ of patients. ${ }^{91025-30}$ In all but the smallest of these, ${ }^{930}$ the percentage of patients achieving clinical remission through enteral nutrition was lower than with corticosteroids. Only in the two largest trials, however, did the difference reach statistical significance. ${ }^{26} 27$ Our recent meta-analysis of randomised controlled trials of enteral nutrition versus corticosteroid treatment showed a treatment benefit for drug therapy compared with nutritional therapy that was not explained merely by intolerance. ${ }^{31}$

Enteral nutrition still constitutes an important therapeutic option, even if less efficacious than corticosteroids. No placebo controlled trials of enteral nutrition have been conducted. Clinical response rates to placebo, however, in active Crohn's disease in controlled clinical trials of drug treatment have ranged from $18 \%$ to $42 \%$ after intervals of 14 to 17 weeks. ${ }^{1232}$ Comparison with observed response rates to exclusive enteral nutrition in prospective studies $^{91025-30}$ suggests that it is effective. Moreover, a reduction in gastrointestinal protein loss, a decrease in intestinal permeability, and a reduction in faecal excretion of indium labelled leucocytes have each been shown, ${ }^{33-35}$ suggesting a direct effect on intestinal inflammation.

Acute Crohn's disease isolated to the colon proved comparatively refractory to treatment with exclusive enteral nutrition, but the small number of such patients precludes any definite conclusion. Adult patients with Crohn's colitis also responded poorly to enteral nutrition in another retrospective report. ${ }^{36}$ Prospectively accrued data from randomised controlled trials of enteral nutrition are not often reported in a site specific fashion. ${ }^{9} 1028-30$ The European Cooperative Crohn's Disease Study (ECCDS) could not show a correlation between site of intestinal inflammation and rate of clinical response to enteral nutrition. ${ }^{26} 27$ Over $60 \%$ of a group of 30 patients treated in randomised fashion with either an elemental or a polymeric liquid diet had inflammation confined to the colon, but the response rate overall was $70 \%{ }^{5}$ Hence prospective studies do not support restriction of enteral nutritional treatment to patients with active small intestinal inflammation.

We have been impressed that young patients adapt to nocturnal nasogastric tube feeding better than would originally have been expected. With instruction and encouragement provided by experienced nursing staff, intolerance was infrequent in the acute phase of treatment. Reported intolerance among predominantly adults in clinical trials versus corticosteroids is $21 \%$ overall, but is much greater when oral administration of formulated food is initially attempted than when, as in our study, the nasogastric route is used from the outset. ${ }^{31}$ The results of our questionnaire show that enteral nutrition is accompanied by frequent adverse effects such as night-time wakening, nausea, loose stools, and vomiting. However, the beneficial effects on disease activity, the improved nutritional state, and improved linear growth make enteral nutrition acceptable to young patients despite these inconveniences.

In the long term, allowing normal food at times when family and friends are eating is particularly important in achieving compliance. The findings of this historical cohort study suggest that administration of formulated food is useful in controlling activity of Crohn's disease even when dietary restrictions are not imposed. The efficacy of such supplementary rather than exclusive enteral nutrition in maintaining remission should be further studied in a randomised, controlled fashion.

The Inflammatory Bowel Diseases Clinical Research Program is supported by a grant from The Hospital for Sick Children is supported by a grant from The
Foundation New Initiatives Fund. Dr Wilschanski is supported by Fellowship grants from Janssen Pharmaceutica and The American Physicians Fellowship.

1 Summers RW, Switz DM, Sessions JT, Becktel JM, Best WR, Kern F, et al. National Cooperative Crohn's Disease Study: results of drug treatment. Gastroenterology 1979; 77: 847-69.

2 Malchow H, Ewe K, Brandes JW, Goebell H, Ehms H, Sommer H, et al. European Cooperative Crohn's Disease Study: results of drug treatment. Gastroenterology 1984; 86: $249-66$.

3 Seidman E, Leleiko N, Ament M, Berman W, Caplan D, Evans J, et al. Symposium Report. Nutritional issues in pediatric inflammatory bowel disease. $f$ Pediatr Gastroenterol Nutr 1991; 12: 424-38. 
4 Fernandez-Banares F, Cabre E, Gonzalez-Huix F, Gassull MA. Enteral nutrition as primary therapy in Crohn's disease. Gut 1994; 25: S55-9.

5 Rigaud D, Cosnes J, Le Quintrec Y, Rene E, Gendre JP, Mignon M. Controlled trial comparing two types of enteral nutrition in treatment of active Crohn's disease. Gut 1991; 32: 1492-7.

6 Aiges H, Markowitz J, Rosa J, Daum F. Home nocturnal supplemental nasogastric feedings in growth-retarded adolescents with Crohn's disease. Gastroenterology 1989; 97: 905-10.

7 National Research Council/National Academy of Sciences. Recommended dietary allowances. Washington, DC: Recommended dietary allow
National Academy, 1989.

8 Hyams J, Ferry GD, Mandel FS, Grybowski JD, Kibort TM, Kirschner BS, et al. Development and validation of a pediatric Crohn's disease activity index. $\mathcal{F}$ Pediatr Gastroenterol Nutr 1991; 12: 439-47.

9 Gonzalez-Huix F, de Leon R, Fernandez-Banares F, Esteve $M$, Cabre E, Acero D, et al. Polymeric enteral diets as primary treatment of active Crohn's disease: a prospective steroid controlled trial. Gut 1993; 34: 778-82.

10 Gorard DA, Hunt JB, Payne-James J, Paler KR, Rees RGP, Clark MI et al. Initial response and subsequent course of Crohn's disease treated with elemental diet or prednisolone. Gut 1993; 34: 1198-202.

11 Harries AD, Jones LA, Danis V, Fifield R, Heatley RV, Newcombe RG, et al. Controlled trial of supplemented Newcombe RG, et al. Controlled trial of supplemented

12 Belli DC, Seidman E, Bouthiller L, Weber AM, Roy CC, Plentincx $M$, et al. Chronic intermittent elemental diet improves growth failure in children with Crohn's disease. Gastroenterology 1988; 94: 603-10.

13 Polk DB, Hattner JT, Kerner JA Jr. Improved growth and disease activity after intermittent administration of a defined formula in children with Crohn's disease. $\mathcal{f}$ Parenteral Enteral Nutr 1992; 16: 499-504.

14 Ruuska T, Savilahti E, Maki M, Ormala T, Visokorpi JK. Exclusive whole protein enteral diet versus prednisolone in the treatment of acute Crohn's disease in children. $\mathcal{F}$ Pediatr Gastroenterol Nutr 1994; 19: 175-80.

15 Park HR, Galloway A, Danesh JZD, Russell RI. Doubleblind controlled trial of elemental and polymeric diets as primary therapy in active Crohn's disease. Eur $\mathcal{f}$ primary therapy in active Crohn's

16 Raouf AH, Hildrey V, Daniel J, Walker RJ, Krasner N, Elias $\mathrm{E}$, et al. Enteral feeding as a sole treatment for Crohn's disease: controlled trial of whole protein versus amino acid based feed. Gut 1991; 32: 702-7.

17 Greenberg GR, Fleming CR, Jeejeebhoy KN, Rosenberg IH, Sales D, Tremaine WJ. Controlled trial of bowel res and nutritional support in the management of Crohn's disease. Gut 1988; 29: 1309-15.

18 Royall D, Jeejeebhoy KN, Baker JP, Allard JP, Habal FM, Cunnane SC, et al. Comparison of amino acid v peptide based enteral diets in active Crohn's disease: clinical and nutritional outcome. Gut 1994; 35: 783-7.

19 Griffiths AM, Nguyen P, Macmillan J, Smith C, Sherman $P$. Growth and clinical course of children and adolescents P. Growth and clinical course of children and
with Crohn's disease. Gut 1993; 34: 939-43.

20 Kelts DG, Grand RJ, Shen G, Watkins JB, Wenlen SH, Boehme C. Nutritional basis of growth failure in children and adolescents with Crohn's disease. Gastroenterology 1979; 76: 720-7.
21 Kirschner BS, Klich JR, Kalmann SS, de Favaro MV, Rosenger IH. Reversal of growth retardation in Crohn's disease with therapy emphasizing oral nutritional restitution. Gastroenterology 1981; 80: 10-5.

22 Griffiths AM, Drobnies A, Soldin SJ, Hamilton JR. Enteric protein loss measured by fecal alpha-1-antitrypsin clearance in the assessment of Crohn's disease activity. $f$ Pediatr Gastroenterol Nutr 1986; 5: 907-11.

23 Motil KJ, Grand RJ, Davis-Kraft L, Ferlic LL, O'BrianSmith E. Growth failure in children with inflammatory bowel disease: a prospective study. Gastroenterology 1993; 105: 681-91.

24 Grimble RF. Nutrition and cytokine action. Nutrition Res Rev 1990; 2: 193-210.

25 Seidman EG, Griffiths AM, Jones A and Issenman R for the Canadian Paediatric Crohn's Disease Study Group. Semielemental diet versus prednisone in the treatment of active Crohn's disease in children and adolescents. Gastroenterology 1993; 104: A778.

26 Lochs H, Steinhardt HJ, Klaus-Wentz B, Zeitz $M$ Vogelsang $\mathrm{H}$, Sommer $\mathrm{H}$, et al. Comparison of enteral nutrition and drug treatment in active Crohn's disease. Results of the European Cooperative Crohn's Disease Study IV. Gastroenterology 1991; 101: 881-8.

27 Malchow H, Ewe K, Brandes JW, Goebell H, Ehms H, Sommer H, et al. European Cooperative Crohn's Disease Study: results of drug treatment. Gastroenterology 1984; 86: $249-66$.

28 Lindor KD, Fleming R, Burnes JU, Nelson JK, Ilstrup DM A randomised prospective trial comparing a defined formula diet, corticosteroids, and a defined formula diet plus corticosteroids in active Crohn's disease. Mayo Clin Proc 1992; 67: 328-33.

29 O'Morain C, Segal AW, Levi AJ. Elemental diet as primary treatment of acute Crohn's disease: a controlled trial. $B M \mathcal{F} 1984$; 288: 1859-62.

30 Seidman EG, Lohoues MJ, Turgeon J, Bouthillier L, Morin CL. Elemental diet versus prednisone as initial therapy in Crohn's disease: early and long term results. Gastroenterology 1991; 100: 250A.

31 Griffiths AM, Ohlsson A, Sherman PM, Sutherland LR Meta-analysis of enteral nutrition as primary treatment of active Crohn's disease. Gastroenterology 1995; 108: active Cro

32 Singleton JW, Hanauer SB, Gitnick GL, Peppercorn MA Robinson MG, Wruble LD, et al. Mesalamine capsules for the treatment of active Crohn's disease: results a 16week trial. Gastroenterology 1993; 104: 1293-301

33 Logan RFA, Gillon J, Ferrington C, Ferguson A Reduction of gastrointestinal protein loss by elemental diet in Crohn's disease of the small bowel. Gut 1981; 22: 383-7.

34 Sanderson IR, Boulton P, Menzies I, Walker-Smith JA. Improvement of abnormal lactulose/rhamnose permeability in active Crohn's disease of the small bowel by an elemental diet. Gut 1987; 28: 1073-6.

35 Teahon $K$, Smethurst $P$, Pearson $M$, Levi AJ, Bjarnason I. The effect of elemental diet on permeability and inflammation in Crohn's disease. Gastroenterology 1991; 101: 84-9.

36 Teahon K, Bjarnason I, Levi AJ. Elemental diets in the management of Crohn's disease: a ten year review. Gut $1990 ; 31: 1133-7$. 